\title{
ENSEÑANZA DE LOS CONCEPTOS QUIMICOS ELEMENTO, COMPUESTO Y MEZCLA: UNA PROPUESTA DESDE EL MODELO DE ALFABETIZACION CIENTÍFICA ${ }^{(1)}$
}

\author{
Andrés Felipe Méndez. ${ }^{(2)} \quad$ zeratul_89@hotmail.com.
}

\section{Resumen}

\begin{abstract}
Este trabajo hace referencia al diseño e implementación de prácticas de laboratorio, desde el modelo de alfabetización científica para la enseñanza de los conceptos químicos: elemento, compuesto y mezcla, a estudiantes de educación media. Se diseñan dos prácticas de laboratorio, además de actividades complementarias para las temáticas de gases y soluciones; se plantean un test y retest con el fin de dar cuenta del trabajo realizado. En las prácticas de laboratorio se incluyen experimentos cotidianos asociados con los conceptos químicos mencionados.

Se establece que, con la aplicación del modelo de alfabetización científica, los estudiantes relacionan mejor los contenidos trabajados y reconocen, durante su desarrollo los conceptos elemento, compuesto y mezcla.
\end{abstract}

\section{Palabras clave}

Alfabetización científica Elemento, compuesto, mezcla, gases, soluciones.

\footnotetext{
(1) Proyecto desarrollado en el Instituto Pedagógico Nacional IPN

${ }^{(2)}$ Estudiante del Departamento de Química de la UPN
} 


\section{INTRODUCCIÓN}

Al iniciarse el siglo XXI nos encontramos en una sociedad que depende cada vez más de sus avances científicos y tecnológicos. La modernidad trae consigo el hecho de que cada vez más la ciencia afecta la vida cotidiana; esta influencia se observa en la creciente demanda de conocimiento científico y tecnológico para tomar decisiones comunes. La ciencia y la tecnología han dejado de ser parte del discurso de unos pocos académicos para formar parte de la vida de los humanos.

Paradójicamente, la mayoría de los miembros de nuestras sociedades -ya sean las autollamadas desarrolladas o las que son consideradas en vías de desarrollo- entienden muy poco acerca de la naturaleza particular de la ciencia que ha transformado el mundo moderno (Jenkins, 1997; Layton, 1994a).

La falta de conocimiento científico útil para que los estudiantes y futuros ciudadanos comprendan y transformen la realidad que les rodea, es parte de una problemática mayor la "alfabetización científica". En los años ochenta se llevaron a cabo, en los Estados Unidos de América del Norte, Inglaterra y otros países, estudios para determinar el nivel de alfabetización científica de la población (Miller, 1983). Los resultados muestran que, en general, la población es iletrada en lo que corresponde a conocimiento científico. Aunque las mismas preguntas que son utilizadas para medir el nivel de alfabetización Científica, son motivo de debate -pues parecen medir sólo memorización de eventos o nombres de científicos más que la comprensión de conocimientos-. Estudios realizados con más refinamiento metodológico, muestran que, a pesar de años de estudio, en general los estudiantes tienen problemas para comprender la ciencia que se les enseña (Driver, Guesne y Tiberghien, 1985; Novak, 1987).
En los últimos años, uno de los mayores desafíos a los que se han tenido que enfrentar los docentes de la educación media, es promover el interés del alumnado por aprender las disciplinas científicas. Durante décadas se consideró que una persona alfabetizada era aquella que sabía leer, escribir y contar. Hoy día sin embargo, resulta indispensable incluir competencias científicas y tecnológicas a la alfabetización básica que aseguren que los ciudadanos puedan desempeñarse con éxito. El proceso de alfabetización científica supone el aprendizaje de la ciencia para que los alumnos adquieran estrategias que les permitan, no sólo incorporar saberes, sino estar en condiciones de profundizar y ampliar el campo de conocimientos durante toda su vida.

\section{DESCRIPCION DEL PROBLEMA}

Una de las principales dificultades que se evidencia en los estudiantes de grado decimo del Instituto Pedagógico Nacional (IPN), con respecto a la asignatura de química, es la falta de motivación por aprenderla, esto se debe, entre otros factores, a que no encuentran relación alguna entre esta y su cotidianidad, se cree que posiblemente esta problemática se fundamenta en el desconocimiento de conceptos científicos que tengan relación con su entorno, conceptos que les permitan elucidar y concretar una interpretación adecuada del mismo, tomando como base modelos que ofrece la ciencia.

Por este motivo, se ha decidido fortalecer en los estudiantes los conceptos de: elemento, compuesto y mezcla, los que se consideran fundamentales para la química y que tienden a ser confundidos entre sí; de este modo se busca determinar, si los estudiantes del grado decimo reconocen adecuadamente las diferencias entre dichos conceptos y consecuentemente aplicar una estrategia de enseñanza- aprendizaje basada en prácticas de laboratorio y enmarcadas en el modelo de alfabetización científica actual. 


\section{MARCO TEORICO}

De acuerdo con el Centro Nacional de Estadísticas de Educación, (EE.UU) "la alfabetización científica es el conocimiento y la comprensión de conceptos científicos y procesos necesarios para la toma de decisiones personales, la participación en asuntos cívicos y culturales, y la productividad económica".

La persona con conocimientos científicos básicos posee la capacidad para:

- La persona con conocimientos científicos básicos posee la capacidad para:

- Entender los hechos científicos básicos y su significado.

- Preguntar, encontrar o determinar respuestas a preguntas derivadas de la curiosidad acerca de las experiencias cotidianas.

- Describir, explicar y predecir fenómenos naturales.

- Realizar lecturas con la comprensión de artículos sobre la ciencia en la prensa popular y participar en la conversación social acerca de la validez de las conclusiones.

- Determinar las cuestiones científicas se basan las decisiones nacionales y locales y las posiciones de expresar que son científicamente y tecnológicamente informado.

- Evaluar la calidad de la información científica sobre la base de su origen y los métodos utilizados para generarla

- Plantear y evaluar los argumentos basados en la evidencia y para aplicar las conclusiones de tales argumentos adecuadamente

Aunque no hay un acuerdo universal sobre cómo medir la alfabetización científica, entidades como la Organización para la Cooperación y el Desarrollo, han tratado de construir correlaciones útiles. En la Ciencias de la Educación Nacional de Normalización, los estándares de contenido definen la alfabetización científica como:
Un proceso de "investigación orientada" que, superando el reduccionismo conceptual, permita a los alumnos participar en la aventura científica de enfrentarse a problemas relevantes y reconstruir los conocimientos científicos, que habitualmente la enseñanza transmite ya elaborados, lo que favorece el aprendizaje más eficiente y significativo.

El concepto de alfabetización científica, muy aceptado hoy en día, cuenta ya con una tradición que se remonta, al menos, a finales de los años 50 , pero es sin duda, durante la última década, cuando esa expresión adquiere categoría y repetidamente es utilizada por los investigadores, diseñadores de currículos y profesores de ciencias (Bybee, 1977).

\section{OBJETIVO}

\section{GENERAL}

Diseñar, implementar y evaluar una propuesta de enseñanza-aprendizaje, desde el enfoque de la alfabetización científica y utilizando prácticas de laboratorio, para la enseñanza de los conceptos químicos elemento, compuesto y mezcla.

\section{OBJETIVOS}

\section{ESPECÍFICOS}

- Diseñar e implementar la propuesta a partir de prácticas de laboratorio relacionados con las temáticas de gases y soluciones.

- Caracterizar los conocimientos teóricos que han construido los estudiantes acerca de los conceptos básicos de la propuesta: elemento, compuesto y mezcla.

- Propiciar espacios en los que los estudiantes reconozcan los modelos teóricos de la ciencia aplicables a su cotidianidad

- Determinar si los estudiantes relacionan dichos conceptos con su entorno 


\section{METODOLOGÍA}

La población objeto de estudio está constituída por cuarenta estudiantes de grado décimo de educación media.

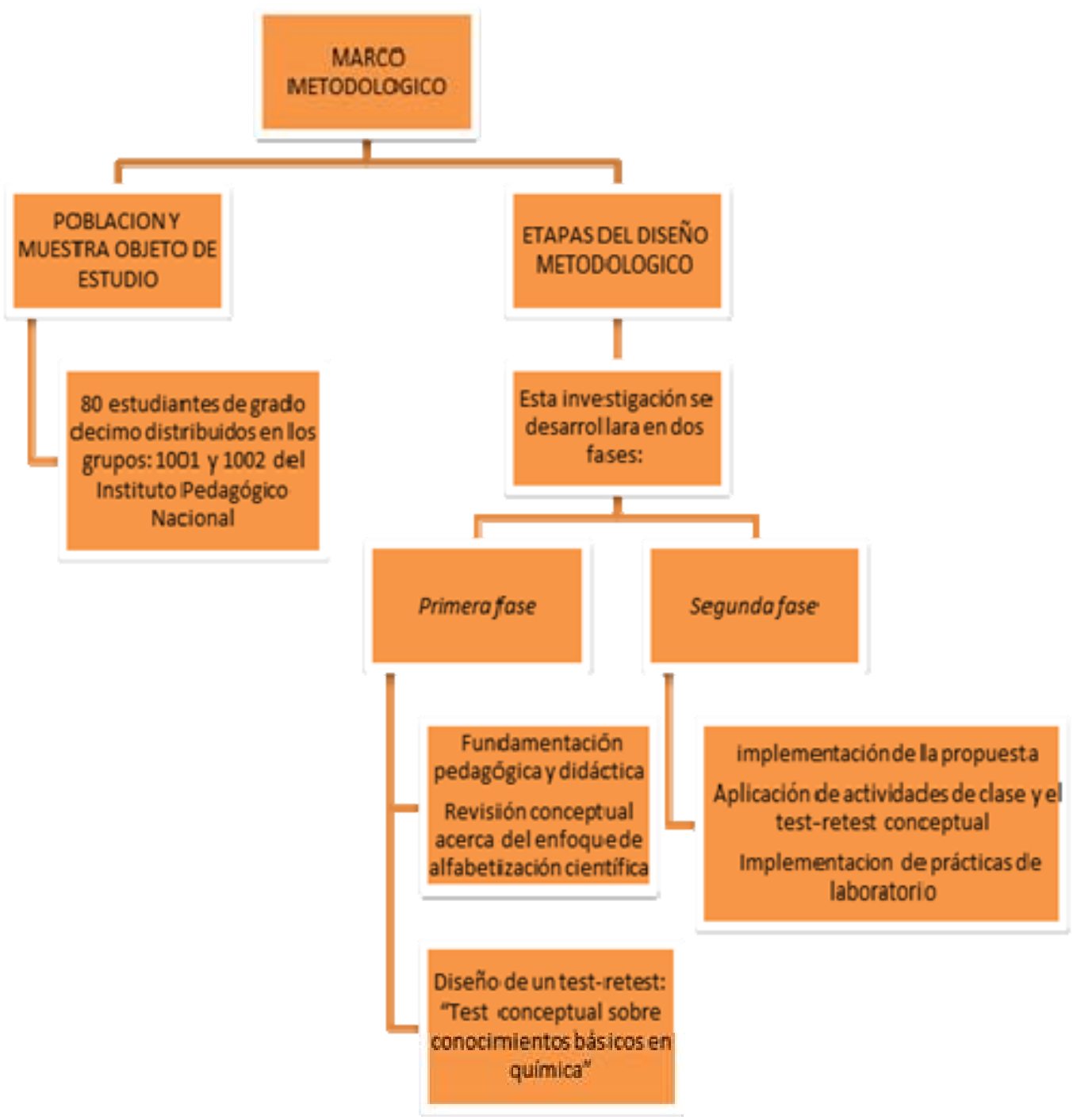


RESULTADOS Y ANALISIS

Primera Fase: Test conceptual conocimientos básicos de química. Anexo 1

\begin{tabular}{|c|c|c|}
\hline \multicolumn{3}{|c|}{ TEST CONCEPTUAL SOBRE } \\
CONOCIMIENTOS BÁSICOS & $\begin{array}{c}\text { DE QUÍMICA } \\
\text { DEN }\end{array}$ \\
\hline $\begin{array}{c}\text { Pregunta } \\
\#\end{array}$ & $\begin{array}{c}\text { Respuestas } \\
\text { correctas }\end{array}$ & $\begin{array}{c}\text { Respuestas } \\
\text { incorrectas }\end{array}$ \\
\hline 1 & 15 & 25 \\
\hline 2 & 5 & 35 \\
\hline 3 & 11 & 29 \\
\hline 4 & 17 & 23 \\
\hline 5 & 29 & 11 \\
\hline 6 & 16 & 24 \\
\hline
\end{tabular}

Tabla 1.Resultados Test conceptual

Los resultados aquí presentados permiten establecer que los estudiantes del grado 1003 presentan inconvenientes para la comprensión de los conceptos de: elemento, compuesto y mezcla, aunque el test propuesto planteaba conceptos ya trabajados, por el grupo de estudiantes, se puede notar que no ha sido un proceso significativo para ellos.

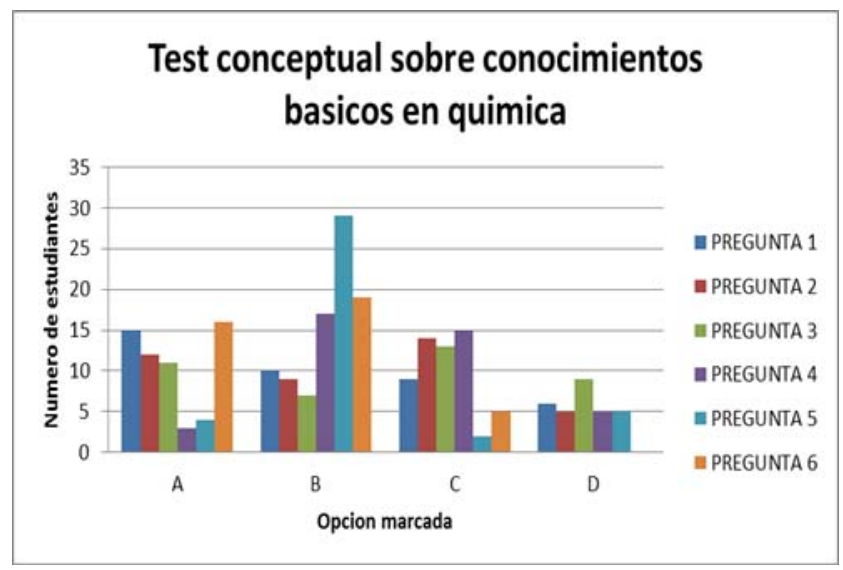

Grafica 1. Resultados test conceptual

Con ayuda de la implementación de una propuesta enmarcada en el enfoque de alfabetización científica, los estudiantes podrán establecer que la ciencia se pude relacionar con su cotidianidad, y de esta forma, se espera que se apropien de los conceptos químicos mencionados.
Segunda fase: visita aula virtual plataforma Educaplus.org

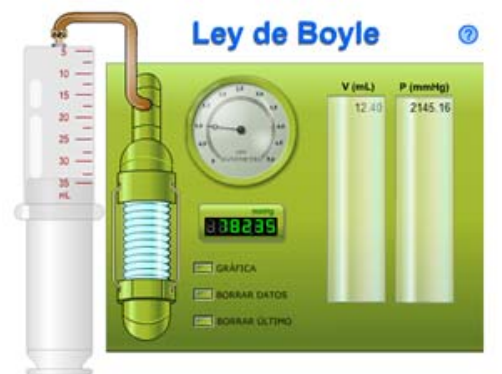

Imagen 1. Práctica virtual- Educaplus.org

Con el objetivo de que los estudiantes realicen una consulta previa acerca de la temática de gases, se precisa que visiten el aula virtual, en donde los estudiantes realizan una consulta interactiva utilizando la plataforma educaplus.org; en esta plataforma, los estudiantes pueden relacionar las leyes de los gases, utilizando simulaciones en las que intervienen, modificando las variables implicadas: volumen, temperatura, presión y número de moles.

\section{Práctica de laboratorio: leyes de los gases}

Se proponen cuatro experimentos dentro de esta práctica, además de los sugeridos por el docente titular.

\section{Experimento 1.}

Tome una cubeta vacía y "pare" una vela en su interior, agregue agua a la cubeta hasta cubrir la mitad de la vela, aproximadamente. Encienda la vela, y después de haber logrado una llama estable, cubra la vela con una probeta, invertida, de modo que esta se sumerja un poco en el agua. ¿Por qué el nivel del líquido asciende? 
Interpretación estudiante. "La llama continúa encendida por algunos segundos, pero debido a la poca disponibilidad de oxigeno (atrapado en el aire dentro de la probeta), se apagará, debido a que ese gas es necesario para la combustión, la cual produce otros gases. Al mismo tiempo, la vela encendida calienta el gas atrapado, lo que provoca que éste se expanda. Al apagarse la vela por falta de oxígeno, la temperatura baja rápidamente y el volumen y la presión de los gases disminuye, lo que provoca que la presión atmosférica externa empuje el agua del plato y esta suba de nivel hasta que se igualen las presiones".

\section{Experimento 2.}

Tome una lata de gaseosa vacía y vierta en ella $10 \mathrm{ml}$ de agua, con ayuda de pinzas para crisol, coloque sobre la llama durante 10 minutos, una vez trascurrido ese tiempo, invierta la lata sobre una vasija con agua fría de tal manera que la lata haga contacto con el agua. ¿Por qué una lata al calentarla previamente e invertirla sobre una cubeta de agua fría, se deforma?

Interpretación estudiante. "El contacto con el agua caliente aumentó la temperatura de la lata, y el aire dentro de ella. Al ponerla en la cubeta de agua fría, el aire interior se va enfriando, su presión disminuirá haciéndose menor que la atmosférica, así que esa diferencia de presión oprimirá el metal, haciendo que la lata se aplaste".

\section{Conclusiones Laboratorio (estudiante)}

$\diamond$ Se afianzan los conocimientos obtenidos en clase, por medio de la práctica en el laboratorio sobre gases $\checkmark$ Se entienden de forma más precisa las leyes de los gases que relacionan presión, volumen y temperatura.

$\checkmark$ Se avanza en cuanto a experiencia con los elementos y aspectos de seguridad que componen una práctica de laboratorio.

\section{Re- Test conceptual}

\begin{tabular}{|c|c|c|}
\hline \multicolumn{3}{|c|}{ Re-TEST CONCEPTUAL SOBRE } \\
CONOCIMIENTOS BASICOS EN QUIMICA \\
\hline $\begin{array}{c}\text { Pregunta } \\
\#\end{array}$ & $\begin{array}{c}\text { Respuestas } \\
\text { correctas }\end{array}$ & $\begin{array}{c}\text { Respuestas } \\
\text { incorrectas }\end{array}$ \\
\hline 1 & 23 & 17 \\
\hline 2 & 21 & 19 \\
\hline 3 & 19 & 21 \\
\hline 4 & 25 & 15 \\
\hline 5 & 32 & 8 \\
\hline 6 & 28 & 12 \\
\hline
\end{tabular}

Tabla 2. Resultados Re-test Conceptual

De acuerdo con los resultados obtenidos en el re -test, se puede determinar que la evolución alcanzada por los estudiantes es considerable; el número de respuestas correctas, comparativamente con las de la primera aplicación del test, se incrementa en un 30\% aproximadamente. Este resultado le confiere validez a la ejecución del proyecto en su totalidad, y hace posible la toma de decisiones, en cuanto al quehacer docente en el aula de clase, desde el modelo de alfabetización científica. Las prácticas de laboratorio diseñadas e implementadas, mediante la utilización de experimentos accesibles a los estudiantes, promueven el interés de estos y determinan, en cierto modo, el aprendizaje efectivo en el aula de clase. 
Re- Test conceptual

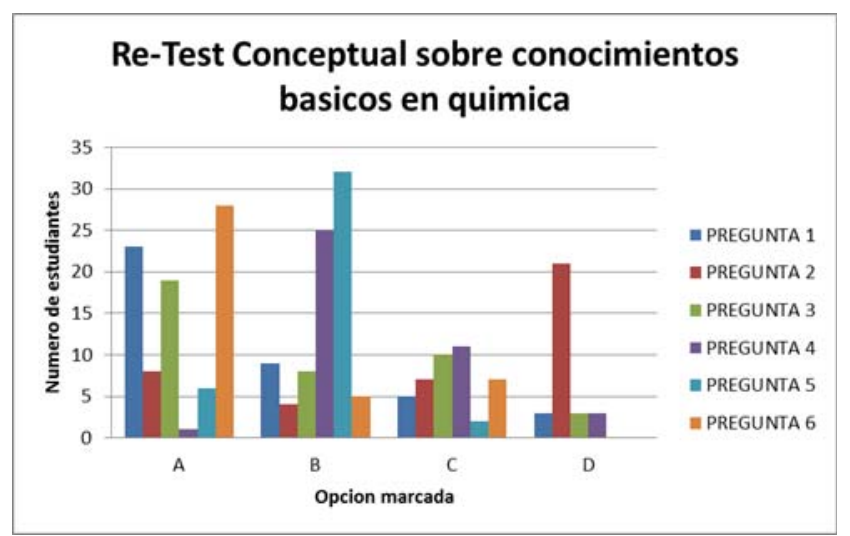

Grafica 2. Resultados Re- test conceptual
En la gráfica anterior se muestran los resultados del re-test conceptual. Se puede observar cómo el número de estudiantes que responden, acertadamente, a las preguntas, es mucho mayor que los que responden de forma incorrecta, y la tendencia a responder correctamente es de aproximadamente un 61\%. Las opciones acertadas sobresalen en la representación gráfica, lo cual se puede definir como un logro en la puesta en marcha de este proyecto

\section{CONCLUSIONES}

$\Theta$ Las actividades empleadas en el desarrollo de la propuesta, y en general, la orientación de las temáticas en el aula de clase, juntamente con las prácticas de laboratorio, permiten afianzar los conceptos básicos aquí planteados.

$\Theta$ El enfoque de alfabetización científica, permite a los estudiantes de grado décimo relacionar los conceptos de elemento, compuesto y mezcla con su cotidianidad, y además de ello, tener una visión más amplia de los procesos que tienen lugar a nivel microscópico y por ende, describir los fenómenos con un mayor grado de acierto.

$\Theta$ La implementación de prácticas de laboratorio, desde el modelo de alfabetización científica, para la enseñanza de la química, promueve en los estudiantes el interés y la motivación por el aprendizaje de la química 


\section{Bibliografía}

^ HODSON, D.(1993). In search of a Rationale for Multicultural Science. Science Education, vol. 77, n.6, pp. 685-711.

^ SOLBES, J. y VILCHES,A. (1997) STS interactions and the teaching of Physics and Chemistry. Science Education, 81, 4, 337-386.

A JENKINS, E. (1997). Towards a functional public understanding of science, en Levinson, R. y Thomas, J. (eds.). Science Today: Problems or Crisis?, pp. 137-150. Londres: Routledge.

A LAYTON, D. (1994). STS in the school curriculum: A movementovertaken by history, en Solomon, J. y Aikenhead, G. (eds.). STS Education International Perspective of Reform, pp. 32-44. Nueva York: Teacher College.

^ JOHNSON, J. (1989). Technology. Report of Project 2061. Phase I. AAAS: Washington DC.

^ MILLER, J. (1983). Scientific literacy: A conceptual and Empirical Review. Daedalus, 112(2), pp. 29-48.

^ ACEVEDO, J. A., VÁZQUEZ, A., MANASSERO, M. A. y ACEVEDO, $P .,(2003)$, Creencias sobre la tecnología y sus relaciones con la ciencia, Revista Electrónica de Enseñanza de las Ciencias, vol. 2, (3), artículo 9.

^ HODSON, D. (1992). In search of a meaningful relationship: an exploration of some issues relating to integration in science and science education. International Journal of Science Education, 14(5), 541-566.

$\wedge$ http://www.oei.es/memoriasctsi/mesa4/m04p35.pdf

^ http://www.utp.edu.co/comutp/archivos/ documentos/11314Cajas.pdf

^ http://www.oei.es/catmexico/una alfabetizacion cientifica\%20.pdf

^ http://www.raco.cat/index.php/ensenanza/article/ viewFile/21633/21467 


\section{ANEXOS \\ TEST CONCEPTUAL SOBRE CONOCIMIENTOS BÁSICOS \\ EN QUÍMICA}

Tópicos relacionados: aspectos:

Fisicoquímicos de sustancias

Analíticos de sustancias

Analíticos de mezclas

1.- Las partículas representadas en el esquema constituyen:

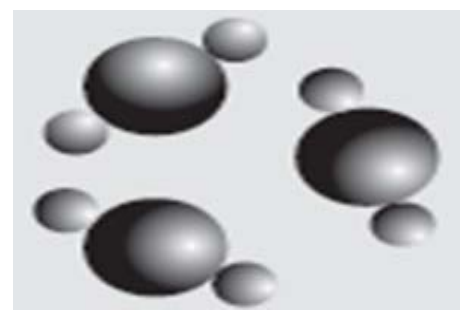

A. Un átomo

B. Un elemento

C .Un compuesto

D. Una mezcla

2.- La siguiente tabla muestra los valores de densidad de tres sustancias

\begin{tabular}{|l|c|}
\hline \multicolumn{1}{|c|}{ Sustancias } & $\begin{array}{c}\text { Densidad a } 25^{\circ} \mathrm{C} \\
(\mathrm{g} / \mathrm{ml})\end{array}$ \\
\hline Tolueno & 0,87 \\
\hline Acido acrílico & 1,06 \\
\hline Agua & 0,99 \\
\hline
\end{tabular}

Se agregan volúmenes diferentes de cada líquido en cuatro recipientes como se ilustra

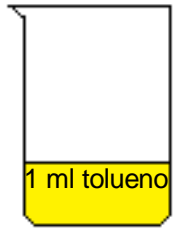

I

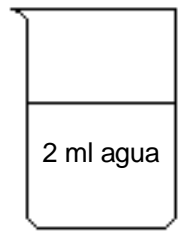

II

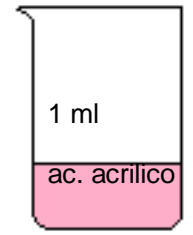

III

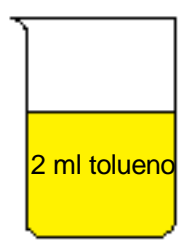

IV

Es válido afirmar que

A. El recipiente IV es el que contiene menor masa.

B. Los recipientes II y IV contienen igual masa.

C. El recipiente III es el que contiene mayor masa.

D. El recipiente III contiene mayor masa que el recipiente I. 


\section{ANEXOS}

3.- $\mathrm{A}$ un tubo de ensayo que contiene agua, se agregan $20 \mathrm{~g}$ de $\mathrm{NaCl}$; se agita la mezcla y se observa que una parte del $\mathrm{NaCl}$ agregado no se disuelve, permaneciendo en el fondo del tubo. Es válido afirmar que en el tubo de ensayo, el agua y el $\mathrm{NaCl}$ constituyen
A. Una mezcla heterogénea
B. Emulsión
C. Una mezcla homogénea
D. Un coloide

4. El esquema muestra el montaje para separar mezclas homogéneas, por medio de la destilación

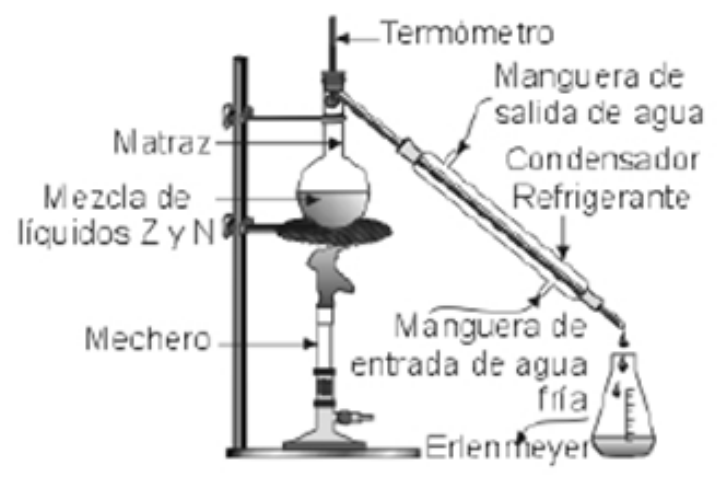

Como se muestra en el esquema, al condensador se encuentran conectadas dos mangueras por donde circula agua fría. Debido a esta corriente de agua, se logra que la temperatura en el condensador sea diferente que la temperatura en el matraz. Esto se realiza con el fin de que la sustancia que proviene del matraz:
A. Reaccione con el agua
B. Se transforme en líquido
C. Aumente su temperatura
D. Se transforme en gas

5. En los recipientes A y B, están contenidas dos sustancias diferentes; A contiene una mezcla de agua y arena y B una mezcla de agua y azúcar; es cierto que:

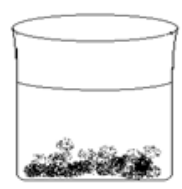

A

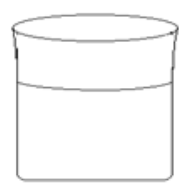

B

1.- A y B contienen mezclas heterogéneas

2.- $A$ contiene una mezcla heterogénea y $B$ una homogénea

3.- $A$ contiene una mezcla homogénea y $B$ una heterogénea

4.- $A$ y $B$ contienen mezclas homogéneas

6. Cuál opción explica mejor el concepto de disolución:

Una mezcla homogénea de dos o más sustancias

Proceso de reducir la concentración de un soluto, habitualmente por adición de mas disolvente Ninguna de las anteriores 